\title{
Sobrevida em dez anos e fatores prognósticos em mulheres com câncer de mama em Joinville, Santa Catarina, Brasil
}

Survival for ten years and prognostic factors for women with breast cancer in Joinville in the State of Santa Catarina, Brazil

${ }^{1}$ Programa de Pós-

Graduação em Nutrição. Universidade Federal de Santa Catarina. R. Eng ${ }^{\circ}$ Max Gerken 66, Glória. 89.216-045 Joinville SC Brasil.alayala@bol.com.br ${ }^{2}$ Policlínica de Atendimento Médico do Boa Vista, Secretaria Municipal de Saúde de Joinville.
Abstract Breast cancer has the highest incidence among women, and reduces survival among female sufferers. This article aims to evaluate the survival and its prognostic factors in women with breast cancer, treated by public sector, in Joinville, Santa Catarina, Brazil. Retrospective cohort study nested in a case-control. The data has been obtained by a questionnaire, by the review of records and death certifications. In the analysis of survival it has been applied the KaplanMeier's statistical method and the Cox's method. 170 women have been evaluated. The survival in ten years was $83.1 \%$ (95\% CI 74.1 to $89.3 \%$ ), and $21(12.4 \%)$ deaths were identified at the time period. The probability of being alive was smaller for those in advanced stages of cancer; the risk of death was higher among those who had another kinds of cancer associated, and among those unsatisfied with life. The death's ratio was 17.1 times bigger among women diagnosed in advanced stages. The variable staging presented higher association with the survival in evaluated women. Detecting the disease early minimizes the mortality by breast cancer.

Key words Breast neoplasms, Neoplasm staging, Survival analysis
Resumo O câncer de mama é o mais incidente no sexo feminino, e reduz a sobrevida das mulheres por ele acometidas. O objetivo deste artigo é avaliar a sobrevida e fatores prognósticos em mulheres com câncer de mama, atendidas no setor público, em Joinville, Santa Catarina, Brasil. Trata-se de estudo de coorte retrospectivo aninhado em um estudo caso-controle. Os dados foram obtidos por meio de questionário, pela revisão dos prontuários e declarações de óbito. Na análise de sobrevida aplicou-se o método estatístico de Kaplan-Meier e o modelo de Cox. Avaliou-se 170 mulheres. A sobrevida em dez anos foi de 83,1\% (IC 95\% 74,1 a 89,3\%), e 21 (12,4\%) óbitos foram identificados no período. A probabilidade de estar viva foi menor para aquelas em estádios avançados. O risco de mortalidade foi maior entre aquelas que tiveram outros tipos de câncer associados e estavam insatisfeitas com a vida. Após análise ajustada o estadiamento clínico foi a única variável que permaneceu associada à sobrevida. A razão de risco de morte foi 17,1 vezes maior entre as mulheres diagnosticadas em estádios avançados. O estadiamento da doença foi a variável com maior associação com a sobrevida das mulheres avaliadas. Detectar a doença precocemente minimiza a mortalidade por câncer de mama.

Palavras-chave Neoplasias da mama, Estadiamento de neoplasias, Análise de sobrevida 


\section{Introdução}

O câncer é uma das maiores causas de mortalidade e morbidade no mundo, com mais de dez milhões de casos novos e mais de seis milhões de mortes por ano. O câncer de mama é o mais incidente entre as mulheres. No Brasil, o câncer de mama é o mais prevalente no sexo feminino, acometendo mulheres com idades entre 40 e 69 anos, sendo a maior causa de morte por câncer ${ }^{1}$.

Na Região Sudeste, o câncer de mama é o mais incidente entre as mulheres, com um risco estimado de 69 casos novos por 100 mil. Sem considerar os tumores de pele não melanoma, este tipo de câncer também é o mais frequente nas mulheres das regiões Sul (65/100.000), Centro-Oeste (48/100.000) e Nordeste (32/100.000). Na Região Norte é o segundo tumor mais incidente $(19 / 100.000)^{2}$.

A informação a respeito de sobreviventes de câncer tem aumentado nas últimas décadas, principalmente nos países de alta renda. Assim como também, o número de pessoas vivendo com essa enfermidade. O número total de registros de casos de sobreviventes de câncer em 2002 era estimado em aproximadamente 25 milhões, e para 2050 a estimativa é de cerca de 70 milhões. Este acréscimo é parcialmente explicado pelo aumento na prevalência geral de câncer, em uma população mundial que está também crescendo. Por outro lado, programas de detecção de tipos comuns de câncer estão identificando muito mais casos, geralmente em estágios relativamente mais precoces ${ }^{3}$.

O câncer de mama, quando diagnosticado em fases iniciais, tem grandes chances de cura, com uma sobrevida de $97 \%$ em cinco anos. A sobrevida é o parâmetro mais utilizado para avaliar resultados na área oncológica, inclusive epidemiológica, na qual as taxas de mortalidade em séries históricas são de alta relevância analítica, sendo possível abordar técnicas estatísticas de análise de sobrevida com observações obtidas em registros de serviços de saúde ${ }^{4}$.

As taxas de sobrevida variam de pessoa para pessoa, esta desigualdade, está relacionada ao curso clínico da doença, que é determinado por uma série complexa de fatores, denominados fatores prognósticos ${ }^{5}$. Estes, para a sobrevida das mulheres com câncer de mama passam por situações designadas como condições de risco, que incluem desde o estadiamento da doença e a idade da paciente no momento do diagnóstico, acesso aos serviços de saúde, condições socioeconômicas e características tumorais, até o apareci- mento de recidiva locorregional após o tratamento por câncer ${ }^{5-8}$.

Apesar de alguns autores sugerirem que fatores prognósticos, como a idade e as situações de recidiva, ainda não tenham comprovado seu impacto em relação à sobrevida das mulheres com câncer de mama, sendo os fatores prognósticos considerados clássicos (estadiamento, tipo e grau histológico do tumor) os que proporcionam provas suficientes para estabelecer a correlação entre piora ou melhora na sobrevida; outros autores referem à importância nas investigações de outros fatores na busca por respostas chaves para a redução da mortalidade por câncer de mama $^{7-10}$.

Diante disso, o objetivo deste trabalho foi investigar os fatores associados à sobrevida em dez anos de mulheres com câncer de mama, de acordo com os fatores prognósticos pertencentes a diferentes recortes temporais incluindo diagnóstico, dados coletados em 2008, dados de tratamento e de mortalidade, disponíveis nos registros de um serviço de referência para diagnóstico e tratamento da doença em Joinville, Santa Catarina.

\section{Metodologia}

Estudo de coorte histórica aninhado em um estudo caso-controle, realizado nos meses de janeiro a julho de 2008, com mulheres de uma unidade de saúde - Posto de Atendimento Médico (PAM) de Boa Vista - considerada centro de referência para tratamento do câncer de mama em Joinville, Santa Catarina.

Joinville está situado na região nordeste do estado de Santa Catarina, com população de 515.250 habitantes em 2010. Município mais populoso de Santa Catarina e o terceiro da região Sul. Sua região metropolitana é constituída pelos municípios adjacentes que formam entre si a Região Metropolitana do Norte/Nordeste Catarinense, com cerca de 1.094.570 habitantes ${ }^{11}$. A região produz 18,9\% do Produto Interno Bruto (PIB) global do estado de Santa Catarina. A cidade é o $3^{\circ}$ maior polo industrial da Região Sul do Brasil ${ }^{12}$. O município possui um dos mais altos índices de desenvolvimento humano (IDH) entre os municípios brasileiros (0.857), ocupando a décima terceira posição nacional, e a quarta entre os municípios catarinenses ${ }^{13}$.

Para o cálculo do tamanho amostral do estudo caso-controle que foi realizado em 2008, foram considerados um nível de confiança de 
95\% e um poder do estudo de $80 \%$ para identificar uma Razão de Odds (RO) de 2,10 dos fatores de risco entre casos e controles, o que indicou 143 casos. Ao resultado foi acrescido um percentual de $20 \%$ para compensar eventuais perdas e/ ou recusas e garantir maior poder ao estudo, o que totalizou 173 casos. Foram consideradas elegíveis para o estudo mulheres que residiam na cidade de Joinville e que haviam descoberto a doença entre os anos de 2000 a 2007. Ao final da pesquisa foram avaliadas 170 mulheres com câncer de mama.

A coleta de dados foi realizada por meio de um questionário que considerou a condição das participantes no momento da entrevista para a maior parte das questões e avaliou as características sociodemográficas e reprodutivas. Características sociodemográficas: faixa etária (até 49 anos; de 50 a 59 anos; 60 anos ou mais); cor (branca; outras: preta/amarela/parda); escolaridade ( 9 ou mais anos; 5 a 8 anos; 0 a 4 anos); e tercis de renda familiar per capita (alto; médio; baixo). Características reprodutivas: idade da menarca (até 11 anos; 12 anos ou mais); aborto (não; sim); e menopausa (sim; não).

Questões relacionadas à saúde também foram incluídas: peso e estatura autorreferidos para avaliação do estado nutricional: eutrofia (índice de massa corporal - IMC de $18,5 \mathrm{~kg} / \mathrm{m}^{2}$ a $24,9 \mathrm{~kg} /$ $\mathrm{m}^{2}$ ); sobrepeso (índice de massa corporal - IMC de $25,00 \mathrm{~kg} / \mathrm{m}^{2}$ a $29,9 \mathrm{~kg} / \mathrm{m}^{2}$ ); obesidade (IMC de $30,00 \mathrm{~kg} / \mathrm{m}^{2}$ ou mais $)^{14}$; tabagismo pregresso (não; sim); incapacidade física (não; sim); doença crônica (não; sim); tensão psicológica (não/ um pouco; sim/bastante); satisfação com a vida (sim; não); autoavaliação de saúde (ótima/boa; regular; ruim/péssima); apoio financeiro (sim; não); apoio social (sim; não); e, outro tipo de câncer (não; sim). E características referentes ao diagnóstico e tratamento após a descoberta da doença: estadiamento (Ts0 e EC I; EC II; EC III e EC IV); tratamento inicial (setorectomia; mastectomia simples; mastectomia radical; quimioterapia ou radioterapia); quimioterapia (não; sim); cirurgia (não; sim); radioterapia (não; sim).

O estadiamento das mulheres foi baseado na classificação TNM proposta em 1988 pela Union for International Cancer Control ${ }^{15}$. A classificação considera o tamanho do tumor, o comprometimento dos linfonodos e a presença ou não de metástase no diagnóstico da doença.

A informação sobre o estadiamento, tratamento e os óbitos das 170 pacientes foi obtida a partir dos dados enviados pela Vigilância Epidemiológica do município para o serviço de saúde, e da análise dos prontuários disponíveis no serviço. Apenas quando a causa do óbito é câncer de mama, as declarações são encaminhadas para o PAM. Pacientes que foram a óbito por causas não relacionadas ao câncer de mama ou a seu tratamento foram censuradas na data de óbito.

Para a análise de sobrevida foram considerados como falha os óbitos (data do óbito) em decorrência do câncer de mama ou consequência do tratamento. Fizeram parte da análise de sobrevida, as pacientes que permaneceram vivas até 31 de julho de 2011.

Foi analisada a sobrevida das pacientes em 120 meses (10 anos). Os dados de sobrevida foram estratificados segundo categorias das variáveis exploratórias, por meio do modelo de Kaplan-Meier ${ }^{16}$.Variáveis de diferentes recortes temporais foram analisadas: diagnóstico, estudo caso-controle de 2008 e mortalidade. O teste de log-rank foi empregado para comparar as funções de sobrevida para cada variável. Razões de risco brutas e ajustadas foram obtidas por meio da Regressão de $\operatorname{Cox}^{17}$. Variáveis com valores de $\mathrm{p} \leq 0,25$ foram inseridas no modelo de análise multivariável. Foram consideradas significativas as associações no nível de significância de 5\%. Para as análises foi utilizado o programa Stata versão 10 .

O estudo caso-controle foi submetido e aprovado pelo Comitê de Ética em Pesquisa da Universidade do Vale do Itajaí (CEP/Univali). Para o estudo longitudinal obteve-se nova aprovação. As mulheres que fizeram parte dessa pesquisa assinaram um termo de consentimento por escrito livre e devidamente esclarecido e a identidade das mesmas foi protegida durante e após o processo de coleta dos dados e divulgação dos resultados.

\section{Resultados}

Foram obtidos dados das 170 mulheres. A cor de pele branca foi a predominante entre as avaliadas $(91,2 \%)$, que possuíam em geral baixa escolaridade $(75,9 \%$ com menos de nove anos de estudo). A maioria tinha mais de 50 anos quando a doença foi diagnosticada $(55,6 \%)$, principalmente no estadiamento II $(53,9 \%)$ (Tabela 1$)$.

O tempo de acompanhamento do estudo variou de 13 a 193 meses, totalizando 13.262 pessoas/ano. A sobrevida em dez anos foi de $83,1 \%$ (IC $95 \% 74,1$ a $89,3 \%)$, sendo que $21(12,4 \%)$ óbitos foram identificados no período avaliado. As mulheres com até 49 anos de idade apresenta- 
Tabela 1. Razão de risco de mortalidade entre mulheres com câncer de mama. Joinville, Santa Catarina, Brasil, $2001-2011$.

\begin{tabular}{|c|c|c|c|c|}
\hline $\begin{array}{c}\text { Variáveis } \\
\text { Categorias }\end{array}$ & n (\%) & $\begin{array}{l}\text { Óbitos } \\
\text { n (\%) }\end{array}$ & $\begin{array}{c}\text { Sobrevida } \\
120 \text { meses (\%) }\end{array}$ & $\begin{array}{c}\text { Teste log-rank } \\
\text { (valor de p) }\end{array}$ \\
\hline Faixa etária diagnóstico & & & & 0,157 \\
\hline Até 49 anos & $75(44,4)$ & $13(17,3)$ & $0,77(0,62 ; 0,87)$ & \\
\hline $50-59$ anos & $56(33,1)$ & $5(8,9)$ & $0,87(0,69 ; 0,95)$ & \\
\hline 60 ou mais & $38(22,5)$ & $3(7,9)$ & $0,92(0,77 ; 0,97)$ & \\
\hline Cor da pele & & & & 0,276 \\
\hline Branca & $155(91,2)$ & $18(11,6)$ & $0,84(0,74 ; 0,90)$ & \\
\hline Outras & $15(8,8)$ & $3(20,0)$ & $0,77(0,44 ; 0,92)$ & \\
\hline Escolaridade & & & & 0,552 \\
\hline 0-4 anos & $90(52,9)$ & $15(16,7)$ & $0,86(0,73 ; 0,93)$ & \\
\hline 5-8 anos & $39(22,9)$ & $2(5,1)$ & $0,82(0,64 ; 0,92)$ & \\
\hline 9 ou mais anos & $41(24,1)$ & $4(9,8)$ & $0,79(0,53 ; 0,92)$ & \\
\hline Tercis de renda familiar per capita & & & & 0,606 \\
\hline Baixo & $54(31,8)$ & $8(14,8)$ & $0,81(0,63 ; 0,91)$ & \\
\hline Médio & $58(34,1)$ & $8(13,8)$ & $0,80(0,59 ; 0,90)$ & \\
\hline Alto & $58(34,1)$ & $5(8,6)$ & $0,89(0,74 ; 0,96)$ & \\
\hline Idade menarca & & & & 0,928 \\
\hline Até 11 anos & $34(20,0)$ & $7(5,1)$ & $0,85(0,77 ; 0,91)$ & \\
\hline 12 ou mais & $136(80,0)$ & $14(10,3)$ & $0,76(0,43 ; 0,91)$ & \\
\hline Aborto & & & & 0,574 \\
\hline Não & $105(61,8)$ & $15(14,3)$ & $0,82(0,70 ; 0,90)$ & \\
\hline Sim & $65(38,2)$ & $6(9,2)$ & $0,85(0,68 ; 0,93)$ & \\
\hline Menopausa & & & & 0,372 \\
\hline Sim & $145(85,3)$ & $17(11,7)$ & $0,84(0,74 ; 0,90)$ & \\
\hline Não & $25(14,7)$ & $4(16,0)$ & $0,84(0,62 ; 0,94)$ & \\
\hline Classificação IMC* & & & & 0,565 \\
\hline Eutrofia & $48(28,2)$ & $7(14,6)$ & $0,82(0,65 ; 0,91)$ & \\
\hline Sobrepeso & $72(42,4)$ & $10(13,9)$ & $0,83(0,68 ; 0,91)$ & \\
\hline Obesidade & $50(29,4)$ & $4(8,0)$ & $0,84(0,58 ; 0,95)$ & \\
\hline Fumo pregresso & & & & 0,566 \\
\hline Não & $112(65,9)$ & $15(13,4)$ & $0,84(0,75 ; 0,90)$ & \\
\hline Sim & $58(34,1)$ & $6(10,3)$ & $0,81(0,59 ; 0,92)$ & \\
\hline Incapacidade física & & & & 0,351 \\
\hline Não & $57(33,5)$ & $5(8,8)$ & $0,91(0,79 ; 0,96)$ & \\
\hline Sim & $113(66,5)$ & $16(14,2)$ & $0,79(0,67 ; 0,88)$ & \\
\hline Doença crônica & & & & 0,975 \\
\hline Não & $90(52,9)$ & $11(12,2)$ & $0,85(0,72 ; 0,92)$ & \\
\hline Sim & $80(47,1)$ & $10(12,5)$ & $0,82(0,66 ; 0,91)$ & \\
\hline
\end{tabular}

ram a pior taxa de sobrevida (77\%); seguidas das mulheres com idades entre 50 a 59 anos (87\%), e com 60 ou mais anos de idade (92\%). A probabilidade de estar viva após dez anos foi menor para aquelas diagnosticadas em estadios III e IV. Entre as mulheres com outro tipo de câncer, o risco de mortalidade foi 3,5 vezes maior em relação às mulheres que tiveram somente $o$ câncer de mama. Entre aquelas insatisfeitas com a vida, a sobrevida foi 2,55 vezes menor quando comparadas à categoria de referência. As mulhe- res que iniciaram o tratamento com quimioterapia ou radioterapia apresentaram 4,32 vezes maior risco de morrer em relação às mulheres que iniciaram o tratamento com setorectomia (Tabela 2).

Após análise ajustada, a única variável que permaneceu associada à sobrevida foi o estadiamento clínico. Mulheres diagnosticadas em estádios mais avançados apresentaram risco 17,1 vezes maior de mortalidade, comparadas àquelas em estádios iniciais (Tabela 2). 


\begin{tabular}{|c|c|c|c|c|}
\hline $\begin{array}{l}\text { Variáveis } \\
\text { Categorias }\end{array}$ & n (\%) & $\begin{array}{l}\text { Óbitos } \\
\text { n (\%) }\end{array}$ & $\begin{array}{c}\text { Sobrevida } \\
120 \text { meses (\%) }\end{array}$ & $\begin{array}{l}\text { Teste log-rank } \\
\text { (valor de p) }\end{array}$ \\
\hline Tensão psicológica & & & & 0,108 \\
\hline Não/um pouco & $141(82,9)$ & $15(10,6)$ & $0,85(0,76 ; 0,91)$ & \\
\hline Sim/bastante & $29(17,1)$ & $6(20,7)$ & $0,72(0,43 ; 0,88)$ & \\
\hline Satisfação com a vida & & & & 0,028 \\
\hline Sim & $128(75,3)$ & $12(9,4)$ & $0,88(0,79 ; 0,93)$ & \\
\hline Não & $42(24,7)$ & $9(21,4)$ & $0,70(0,46 ; 0,84)$ & \\
\hline Autoavaliação de saúde & & & & 0,541 \\
\hline Ótima/boa & $104(61,2)$ & $13(12,5)$ & $0,81(0,67 ; 0,89)$ & \\
\hline Regular & $50(29,4)$ & $5(10,0)$ & $0,90(0,77 ; 0,96)$ & \\
\hline Ruim/péssima & $16(9,4)$ & $3(18,8)$ & $0,80(0,50 ; 0,93)$ & \\
\hline Apoio financeiro & & & & 0,425 \\
\hline Sim & $85(50,0)$ & $9(10,6)$ & $0,84(0,7 ; 0,92)$ & \\
\hline Não & $85(50,0)$ & $12(14,1)$ & $0,83(0,71 ; 0,9)$ & \\
\hline Apoio social & & & & 0,440 \\
\hline Sim & $142(85,5)$ & $19(13,4)$ & $0,82(0,72 ; 0,89)$ & \\
\hline Não & $28(16,5)$ & $2(7,1)$ & $0,93(0,73 ; 0,98)$ & \\
\hline Outro tipo de câncer & & & & 0,015 \\
\hline Não & $158(92,9)$ & $17(10,8)$ & $0,87(0,80 ; 0,92)$ & \\
\hline Sim & $12(7,1)$ & $4(33,3)$ & $0,28(0,01 ; 0,70)$ & \\
\hline Estadiamento & & & & $<0,001$ \\
\hline Ts0 e EC I & $49(29,3)$ & $1(2,0)$ & $0,98(0,86 ; 1,00)$ & \\
\hline EC II & $90(53,9)$ & $11(12,2)$ & $0,81(0,67 ; 0,90)$ & \\
\hline EC III e EC IV & $28(16,8)$ & $9(32,1)$ & $0,60(0,34 ; 0,79)$ & \\
\hline Tratamento inicial & & & & 0,192 \\
\hline Setorectomia & $59(35,1)$ & $3(5,1)$ & $0,94(0,81 ; 0,98)$ & \\
\hline Mastectomia simples & $15(8,9)$ & $1(6,7)$ & $0,93(0,61 ; 0,99)$ & \\
\hline Mastectomia radical & $76(45,2)$ & $13(17,1)$ & $0,78(0,64 ; 0,87)$ & \\
\hline Quimioterapia ou radioterapia & $18(10,7)$ & $4(22,2)$ & $0,77(0,50 ; 0,91)$ & \\
\hline Quimioterapia & & & & 0,043 \\
\hline Não & $37(21,8)$ & $1(2,7)$ & $0,97(0,82 ; 1,00)$ & \\
\hline Sim & $133(78,2)$ & $20(15,0)$ & $0,79(0,67 ; 0,87)$ & \\
\hline Cirurgia & & & & 0,108 \\
\hline Não & $2(1,2)$ & $1(50,0)$ & $0,50(0,01 ; 0,91)$ & \\
\hline Sim & $168(98,8)$ & $20(11,9)$ & $0,83(0,74 ; 0,90)$ & \\
\hline Radioterapia & & & & 0,281 \\
\hline Não & $40(23,5)$ & $3(7,5)$ & $0,92(0,78 ; 0,97)$ & \\
\hline Sim & $130(76,5)$ & $18(13,8)$ & $0,80(0,68 ; 0,88)$ & \\
\hline
\end{tabular}

${ }^{*} \mathrm{IMC}=$ Índice de Massa Corporal.

\section{Discussão}

Por meio de um estudo de coorte aninhado em um caso-controle, este estudo objetivou identificar a sobrevida em dez anos e os fatores associados entre mulheres com câncer de mama. Dentre as variáveis investigadas, o estadiamento foi aquela com maior magnitude de associação com a sobrevida das pacientes. Na análise inicial, variáveis como, insatisfação com a vida e presença de outro tipo de câncer além do de mama, estiveram associadas à menor sobrevida.
A sobrevida estimada em dez anos no presente estudo foi de $83,1 \%$. Guerra et al. ${ }^{18}$ encontraram resultados semelhantes aos desta pesquisa, com uma sobrevida de $81,8 \%$ em uma coorte de cinco anos de mulheres com câncer de mama invasivo em Juiz de Fora, Minas Gerais. No entanto, a sobrevida verificada no presente estudo foi superior àquela observada em cinco anos em uma coorte hospitalar de mulheres com carcinoma invasivo do Hospital do Câncer do Instituto Nacional de Câncer (INCA), a qual foi de $75 \%{ }^{6}$. Moraes et al. ${ }^{9}$ numa coorte retrospectiva 
Tabela 2. Análise univariada, sobrevida e razão de risco em mulheres com câncer de mama. Joinville, Santa Catarina, Brasil, 2001 - 2011.

\begin{tabular}{|c|c|c|c|c|}
\hline $\begin{array}{l}\text { Variáveis } \\
\text { Categorias }\end{array}$ & $\begin{array}{l}\text { Razão de Riscos } \\
\text { Bruta (IC 95\%) }\end{array}$ & $\begin{array}{l}\text { Teste Wald } \\
\text { (valor } \mathbf{p})\end{array}$ & $\begin{array}{c}\text { Razão de Risco } \\
\text { Ajustada (IC 95\%) }\end{array}$ & $\begin{array}{c}\text { Teste Wald } \\
\text { (valor } \mathbf{p} \text { ) }\end{array}$ \\
\hline Faixa etária diagnóstico & & 0,149 & & \\
\hline Até 49 anos & 1,00 & & & \\
\hline $50-59$ anos & $0,52(0,19 ; 1,46)$ & & & \\
\hline 60 ou mais & $0,46(0,13 ; 1,62)$ & & & \\
\hline Cor da pele & & 0,285 & & \\
\hline Branca & 1,00 & & & \\
\hline Outras & $1,95(0,57 ; 6,64)$ & & & \\
\hline Escolaridade & & 0,313 & & \\
\hline $0-4$ anos & 1,00 & & & \\
\hline $5-8$ anos & $1,58(0,56 ; 4,46)$ & & & \\
\hline 9 ou mais anos & $1,63(0,58 ; 4,59)$ & & & \\
\hline \multicolumn{5}{|c|}{ Tercis de renda familiar per capita } \\
\hline Baixo & 1,00 & 0,359 & & \\
\hline Médio & $0,94(0,35 ; 2,51)$ & & & \\
\hline Alto & $0,59(0,19 ; 1,79)$ & & & \\
\hline \multicolumn{5}{|l|}{ Idade menarca } \\
\hline Até 11 anos & 1,00 & 0,928 & & \\
\hline 12 ou mais & $0,95(0,32 ; 2,83)$ & & & \\
\hline \multicolumn{5}{|l|}{ Aborto } \\
\hline Não & 1,00 & 0,575 & & \\
\hline Sim & $0,77(0,31 ; 1,91)$ & & & \\
\hline \multicolumn{5}{|l|}{ Menopausa } \\
\hline Sim & 1,00 & 0,372 & & \\
\hline Não & $1,64(0,55 ; 4,88)$ & & & \\
\hline \multicolumn{5}{|l|}{ Classificação IMC* } \\
\hline Eutrofia & 1,00 & 0,384 & & \\
\hline Sobrepeso & $1,02(0,39 ; 2,69)$ & & & \\
\hline Obesidade & $0,56(0,17 ; 1,93)$ & & & \\
\hline \multicolumn{5}{|l|}{ Fumo pregresso } \\
\hline Não & 1,00 & 0,567 & & \\
\hline $\operatorname{Sim}$ & $0,76(0,29 ; 1,95)$ & & & \\
\hline \multicolumn{5}{|l|}{ Incapacidade física } \\
\hline Não & 1,00 & 0,355 & & \\
\hline Sim & $1,61(0,59 ; 4,39)$ & & & \\
\hline \multicolumn{5}{|l|}{ Doença crônica } \\
\hline Não & 1,00 & 0,975 & & \\
\hline Sim & $1,01(0,43 ; 2,39)$ & & & \\
\hline
\end{tabular}

de base hospitalar com pacientes do sexo feminino provenientes de localidades da região central do Rio Grande do Sul, identificaram uma sobrevida em cinco anos de 78,7\%; assim como, Traebert ${ }^{19}$ em uma pesquisa realizada em Florianópolis num serviço de referência de mastologia em mulheres com mais de 65 anos, verificou sobrevida geral em cinco anos de $73,1 \%$; e também, Schneider e D'orsi ${ }^{20}$, em um estudo desenvolvido em Santa Catarina, no qual observaram uma sobrevida de $76,6 \%$ para mulheres com câncer de mama.
Estudo realizado entre as mulheres de Joinville no período compreendido entre 2000 e 2009 encontrou sobrevida de $57,8 \%$ ao final do período de 10 anos, enquanto que em cinco anos a sobrevida estimada foi de $78,6 \%{ }^{21}$. Em função de parte das mulheres avaliadas na presente pesquisa serem parte do grupo investigado por Ayala ${ }^{21}$, é possível sugerir a atuação de um viés de sobrevivência entre elas. Isto é, mulheres vivas no ano do estudo caso-controle (2008), tinham maiores chances de estarem vivas ao final do presente estudo. Além disso, é possível observar menor per- 


\begin{tabular}{|c|c|c|c|c|}
\hline $\begin{array}{l}\text { Variáveis } \\
\text { Categorias }\end{array}$ & $\begin{array}{l}\text { Razão de Riscos } \\
\text { Bruta (IC 95\%) }\end{array}$ & $\begin{array}{c}\text { Teste Wald } \\
\text { (valor } \mathbf{p})\end{array}$ & $\begin{array}{c}\text { Razão de Risco } \\
\text { Ajustada (IC 95\%) }\end{array}$ & $\begin{array}{c}\text { Teste Wald } \\
\text { (valor p) }\end{array}$ \\
\hline Tensão psicológica & & 0,116 & & \\
\hline Não/um pouco & 1,00 & & & \\
\hline Sim/bastante & $2,14(0,83 ; 5,51)$ & & & \\
\hline Satisfação com a vida & & 0,034 & & \\
\hline Sim & 1,00 & & & \\
\hline Não & $2,55(1,07 ; 6,06)$ & & & \\
\hline Autoavaliação de saúde & & 0,663 & & \\
\hline Ótima/boa & 1,00 & & & \\
\hline Regular & $0,79(0,28 ; 2,23)$ & & & \\
\hline Ruim/péssima & $1,76(0,50 ; 6,18)$ & & & \\
\hline Apoio financeiro & & 0,428 & & \\
\hline Sim & 1,00 & & & \\
\hline Não & $1,42(0,6 ; 3,38)$ & & & \\
\hline Apoio social & & 0,446 & & \\
\hline Sim & 1,00 & & & \\
\hline Não & $0,57(0,13 ; 2,44)$ & & & \\
\hline Outro tipo de câncer & & 0,023 & & \\
\hline Não & 1,00 & & & \\
\hline Sim & $3,53(1,19 ; 10,51)$ & & & \\
\hline Estadiamento & & 0,001 & & 0,001 \\
\hline Ts0 e EC I & 1,00 & & 1,00 & \\
\hline EC II & $6,06(0,78 ; 46,95)$ & & $6,06(0,78 ; 46,95)$ & \\
\hline EC III e EC IV & $17,1(2,17 ; 135,05)$ & & $17,1(2,17 ; 135,05)$ & \\
\hline Tratamento inicial & & 0,036 & & \\
\hline Setorectomia & 1,00 & & & \\
\hline Mastectomia simples & $1,46(0,15 ; 14,09)$ & & & \\
\hline Mastectomia radical & $2,93(0,83 ; 10,32)$ & & & \\
\hline Quimioterapia ou radioterapia & $4,32(0,97 ; 19,36)$ & & & \\
\hline Quimioterapia & & 0,077 & & \\
\hline Não & 1,00 & & & \\
\hline Sim & $6,13(0,82 ; 45,73)$ & & & \\
\hline Cirurgia & & 0,143 & & \\
\hline Não & 1,00 & & & \\
\hline Sim & $0,22(0,03 ; 1,66)$ & & & \\
\hline Radioterapia & & 0,289 & & \\
\hline Não & 1,00 & & & \\
\hline Sim & $1,94(0,57 ; 6,58)$ & & & \\
\hline
\end{tabular}

"IMC = Índice de Massa Corporal.

centual de mulheres em estadiamentos mais avançados entre aquelas que fizeram parte do estudo caso-controle anteriormente realizado.

A sobrevida das mulheres avaliadas não diferiu estatisticamente em relação à faixa etária. No entanto, observou-se que as mulheres com até 49 anos de idade apresentaram a pior taxa de sobrevida em 120 meses (77\%). Existem controvérsias a respeito da idade no momento do diagnóstico e o prognóstico da sobrevida para mulheres com câncer de mama, mas, a faixa etária tem sido investigada e evidenciada em muitos estudos como um fator preponderante no melhor prognóstico dessa doença ${ }^{5,9,20}$. Enquanto uma pesquisa encontrou pior sobrevida em mulheres com idade inferior a 30 anos quando comparadas às demais faixas etárias, sendo que aos 57 meses, somente $46,7 \%$ delas permaneciam vivas $^{20}$. Em um estudo realizado pela American Cancer Society ${ }^{22}$ constatou-se que entre os anos de 2000 a 2004, dos casos de câncer diagnosticados e dos casos de óbito por câncer de mama, $95 \%$ e $97 \%$, respectivamente, foram em mulheres com 40 anos ou mais. 
Sabe-se que o estadiamento avançado do câncer de mama está associado a uma pior sobrevi$\mathrm{da}^{23}$. Na presente pesquisa a maior parte das mulheres encontrava-se no estadiamento II $(53,9 \%)$, diferentemente dos resultados constatados na grande maioria das instituições de câncer, os quais apontam que os estadiamentos III e IV chegam a corresponder a cerca de $60 \%$ dos diagnósticos iniciais. Os dados de 1999 a 2003 das unidades I, II e III do Instituto Nacional de Câncer, no Hospital do Câncer, indicaram que as taxas de câncer de mama em estádios avançados (estádios III e IV) estiveram próximas a 55\%, enquanto $35 \%$ encontravam-se no estádio II $^{24}$.

Entretanto, cabe salientar que no Brasil poucos estudos têm examinado as tendências do estadiamento dos casos de câncer de mama ao longo dos anos. Os dados obtidos a partir dos registros hospitalares de câncer mostraram que, na última década, houve redução no percentual de casos de câncer de mama em estádio avançado, apesar de ainda serem superiores aos demais. O estudo com maior casuística realizado no Brasil aponta nesta direção, segundo a pesquisa a maioria dos casos de câncer de mama encontravamse no estádio II $(42,8 \%)$, ao passo que os casos em estádio avançado corresponderam a $45,3 \%{ }^{25}$.

No estudo em questão constatou-se que houve uma redução da probabilidade de sobrevida conforme o aumento do estadiamento (Ts0 e EC I: 98\%; EC II: 81\%; EC III e EC IV: 60\%). Corroborando com estes achados, Moraes et al. ${ }^{9}$ verificaram que as probabilidades de sobrevida em dez anos para as pacientes foram reduzindo de acordo com os estádios (I: 97\%; IIa: 87\%; IIb: 70\%; IIIa+IIIb: 73\% e IV: $0 \%$ ). O mesmo foi encontrado no estudo realizado por Guerra et al. ${ }^{18}$, no qual evidenciou-se uma redução progressiva na sobrevida de acordo com o aumento do estádio da doença (I: 92,7\%; II: 88,3\%; III: 67\%; IV: 54\%).

A maior parte das mulheres avaliadas tinha mais de 50 anos de idade, cor de pele predominantemente branca, e baixa escolaridade. Estes resultados estão em consonância com o achado de outro estudo, demostrando que o câncer de mama é mais frequente em mulheres brancas com mais de 50 anos $^{26}$. Contudo, a associação entre cor da pele e sobrevida entre as mulheres avaliadas não pode ser considerada conclusiva, devido ao reduzido número de mulheres com cor de pele diferente da branca na amostra investigada, que reflete a colonização predominantemente europeia da cidade de Joinville. Em relação à escolaridade, pesquisas apontam que mulheres com menos de oito anos de estudo tendem a realizar mamografia com menor frequência ${ }^{27}$ e a terem maior prevalência de câncer de mama ${ }^{28}$.

No presente estudo, não é possível sugerir que as características socioeconômicas e as questões relacionadas à saúde, como por exemplo, peso, tabagismo pregresso, incapacidade física, entre outras, sejam fatores que possam estar associados a maior ou menor sobrevida das mulheres com câncer de mama, visto que, algumas destas variáveis não estiveram associadas nem mesmo na análise bruta, e outras perderam significância estatística após ajuste para o estadiamento da doença. Ou seja, isso indica que o estadiamento da doença, pode ter sido um fator de confusão na associação entre sobrevida e algumas das variáveis investigadas.

$\mathrm{O}$ risco de morte entre as mulheres que apresentaram outro tipo de câncer, além do câncer de mama neste estudo, foi 3,5 vezes maior quando comparadas às mulheres que tiveram somente $o$ câncer de mama. Pesquisas têm demonstrado tendência de pior sobrevida entre pacientes com câncer de mama e comorbidades graves ${ }^{29-31}$. O estudo de Houterman et al. ${ }^{29}$ indicou que o risco de morte em mulheres com câncer de mama com menos de 70 anos e com comorbidades de alto impacto era 2,9 vezes maior, em relação àquelas sem comorbidades. Mell et al. ${ }^{31}$ verificaram 1,9 vezes maior risco de morte entre mulheres com câncer de mama e comorbidades associadas.

As variáveis tensão psicossocial e autoavaliação de saúde não estiveram estatisticamente associadas com a sobrevida das mulheres com câncer de mama de Joinville. Para a insatisfação com a vida, a associação foi significativa na análise bruta. Autores observaram associações significativas entre a satisfação com diferentes aspectos da vida e a sobrevida por câncer de ovário ${ }^{32}$, pâncreas $^{33}$, colo e reto ${ }^{34}$ e mama ${ }^{35}$.

No estudo que envolveu séries consecutivas de casos de pacientes com câncer de mama, confirmados histologicamente e tratados pelo Centro Americano de Tratamento do Câncer, a satisfação com a saúde e as subescalas físicas do instrumento utilizado para avaliar a qualidade de vida foram estatisticamente associadas com sobrevivência entre as pacientes. A sobrevivência mediana para os escores baixo e alto das escalas foi de 17,8 e 35,3 meses, respectivamente. A satisfação dos pacientes com a saúde, e com as subescalas físicas foram consideradas preditores de sobrevivência, independentemente do estágio da doença ${ }^{35}$.

Estudos demonstram que a adição da quimioterapia e da radioterapia ao tratamento do cân- 
cer de mama promove aumento da sobrevida geral $^{5,36,37}$. Entretanto, nesta pesquisa as pacientes que utilizaram como tratamento inicial do câncer de mama a quimioterapia ou a radioterapia apresentaram maior probabilidade de morrer (RR 4,33 IC 0,97 - 19,36), em relação àquelas que utilizaram a setorectomia. Schneider e D'Orsi ${ }^{20}$ constataram que mulheres que receberam tratamento sem associação com a quimioterapia demonstraram melhor sobrevida $(82,2 \%)$ quando comparadas àquelas com tratamento associado à quimioterapia $(71,7 \%)$. Guerra et al. ${ }^{18}$ verificaram que as mulheres que fizeram uso de quimioterapia e de radioterapia apresentaram sobrevida significativamente desfavorável, com 79,2\% e 79,9\%, respectivamente, em relação àquelas que não utilizaram estas terapias complementares $(87,5 \% \mathrm{e}$ $89 \%$, respectivamente). E Bezerra et al. ${ }^{38}$ demonstraram que o uso de terapias adjuvantes (quimioterapia, radioterapia e hormonioterapia) influenciou negativamente a qualidade de vida de mulheres com câncer de mama. Ressalta-se que as mulheres que iniciaram o tratamento com quimioterapia ou radioterapia, provavelmente apresentavam estádios mais avançados da doença, o que explicaria a perda da significância estatística da variável tratamento inicial quando ajustada para o estadiamento clínico.

Os resultados supracitados podem ser explicados pelas limitações que os bancos de dados secundários podem apresentar, principalmente em relação aos erros de classificação quanto ao primeiro tratamento recebido. Além do uso inadequado das intervenções terapêuticas, indo de encontro aos protocolos clínicos recomendados. Um estudo realizado no Rio de Janeiro com mulheres com câncer de mama verificou que algumas intervenções recomendadas e comprovadamente eficazes foram subutilizadas, enquanto outras que são desaconselhadas acabaram sendo realizadas ${ }^{39}$.

Este estudo apresentou algumas limitações, por se tratar de um estudo de coorte histórica aninhado a um estudo caso controle, pode ter ocorrido perda de dados importantes nos prontuários das pacientes, assim como o subpreenchimento dos mesmos. Além disso, nesta pesquisa avaliaram-se casos prevalentes, o que pode ter causado viés de sobrevivência, visto que qualquer característica associada com a sobrevivência será mais comum entre os casos.
Outra limitação deste estudo se deve ao fato de que a sobrevida de câncer de mama foi investigada de acordo com as características socioeconômicas apenas das mulheres que utilizavam o serviço público de saúde e, portanto, de mais baixa renda, menor escolaridade e menos propensas ao acesso à assistência à saúde ${ }^{40}$. As mulheres que utilizam os serviços privados, com melhor acesso à assistência não foram examinadas, o que impossibilitou estabelecer comparações entre os dois grupos. Pesquisas têm demonstrado disparidades na sobrevida das mulheres com câncer de mama em relação ao nível socioeconômico. De acordo com alguns estudos, mulheres com menor nível socioeconômico apresentam pior sobrevida ${ }^{41-43}$. Além disso, mulheres provenientes de condições socioeconômicas mais desfavoráveis podem ter morrido antes da realização da pesquisa. A outra limitação diz respeito às informações sobre o tratamento, pois foi considerado apenas o tratamento inicial de acordo com o estádio do câncer de mama, as várias combinações das modalidades terapêuticas na sequência do tratamento não foram levadas em conta no estudo.

Em conclusão, os achados deste estudo encontram-se em consonância com os dados da literatura. Estes apontaram que as mulheres em estadiamentos avançados tiveram pior sobrevida em relação àquelas em estadiamentos iniciais. Além disso, a presença de comorbidades e a insatisfação com a vida estiveram associadas à pior sobrevida nas pacientes analisadas, contudo, a perda de associação após ajuste para estadiamento indica que este seja fator de confusão para estas variáveis. O risco aumentado de morte também foi observado nas mulheres mais jovens.

Por fim, considerando a escassa evidência de estudos que confirmem que os fatores psicossociais desempenham um papel importante na sobrevivência das mulheres com câncer de mama, e tendo em mente que os achados deste estudo sugerem que a insatisfação com a vida é um aspecto em potencial para a sobrevida dessas mulheres, seria notável que fossem realizados estudos que utilizassem ferramentas de avaliação válidas, os quais investigassem na cidade de Joinville, o papel deste aspecto na sobrevida das portadoras de câncer de mama. 


\section{Colaboradores}

DA Höfelmann e JC Anjos participaram da concepção e planejamento; obtenção, análise, interpretação dos dados e redação do artigo. DA Höfelmann também realizou a revisão crítica do artigo. AL Ayala participou da análise, interpretação dos dados e redação do artigo.

\section{Referências}

1. Trufelli DC, Miranda VC, Santos MBB, Fraile NMP, Pecoroni PG, Gonzaga SFR, Riechelmann R, Kaliks R, Del Giglio A. Análise do atraso no diagnóstico e tratamento do câncer de mama em um hospital público. Rev Assoc Med Bras 2008; 54(1):72-76.

2. Instituto Nacional de Câncer (Inca). Estimativas 2012: incidência de câncer no Brasil. Rio de Janeiro: Inca; 2011.

3. World Cancer Research Fund, American Institute for Cancer Research (AIRC). Food, nutrition, physical activity, and the prevention of cancer: a global perspective. Washington: AIRC; 2007.

4. Pedersen L, Gunnarsdottir KA, Rasmussen BB, Moeller S, Lanng C. The prognostic influence of multifocality in breast cancer patients. The Breast 2004; 13(3):188-193.

5. Abreu E, Koifman S. Fatores prognósticos no câncer da mama feminino. Rev Bras Cancerol 2002. 48(1):113-131.

6. Mendonça GAS, Silva AM, Caula WM. Características tumorais e sobrevida de cinco anos em pacientes com câncer de mama admitidas no Instituto Nacional de Câncer, Rio de Janeiro, Brasil. Cad Saude Publica 2004; 20(5):1232-1239.

7. Flores-Luna L, Salazar-Martínez E, Duarte-Torres RM, Torres-Mejía G, Alonso-Ruiz P, Lazcano-Ponce E. Factores pronósticos relacionados con la supervivencia del cáncer de mama. Salud Pública Méx 2008; 50(2):119-125.

8. Martins E, Freitas-Junior R, Curado MP, Freitas NMA, Oliveira JC, Silva CMB. Evolução temporal dos estádios do câncer de mama ao diagnóstico em um registro de base populacional no Brasil central. Rev Bras Ginecol Obstet 2009; 31(5):219-223.

9. Moraes AB, Zanini RR, Turchiell MS, Riboldi J, Medeiros LR. Estudo da sobrevida de pacientes com câncer de mama atendidas no hospital da Universidade Federal de Santa Maria, Rio Grande do Sul, Brasil. Cad Saude Publica 2006; 22(10):2219-2228.

10. Rauschecker H, Clarke M, Gatzemeier W, Recht A. Tratamiento sistémico para la recidiva locorregional en mujeres con cáncer de mama (Revisión Cochrane traducida). In: La Biblioteca Cochrane Plus $n^{\circ}$ 4. Oxford: Update Software Ltd.; 2008.

11. Instituto Brasileiro de Geografia e Estatística (IBGE). Primeiros dados do censo 2010. [site na Internet]. [acessado 2012 out 20]. Disponível em: http:// www.censo2010.ibge.gov.br/resultados

12. Instituto Brasileiro de Geografia e Estatística (IBGE). Cidades@ [site na Internet]. [acessado 2012 out 19]. Disponível em: http://www.ibge.gov.br/cidadesat/ default.php

13. Organização das Nações Unidas (ONU). Programa das Nações Unidas para o desenvolvimento. Atlas do desenvolvimento humano. Indice de desenvolvimento humano - municipal, 1991 e 2000. Washington: ONU; 2000.

14. World Health Organization (WHO). Diet, nutrition and prevention of chronic diseases: report of a Joint WHO/FAO Expert Consultation. Geneva: WHO; 1995. (Technical Report Series, n. 916). 
15. Union for International Cancer Control (UICC). TNM Classification of malignant tumours. New York: John Wiley \& Sons INC; 2002.

16. Armitage P, Berry G. Statistical methods in medical research. $2^{\text {nd }}$ ed. Oxford: Oxford Scientific Publications; 1987.

17. Kleinbaum DG. Survival analysis - a self-learning text. New York: Springer Verlag; 1997.

18. Guerra MR, Mendonça GAS, Bustamante-Teixeira MT, Cintra JRD, Carvalho LM, Magalhães LV. Sobrevida de cinco anos e fatores prognósticos em coorte de pacientes com câncer de mama assistidas em Juiz de Fora, Minas Gerais, Brasil. Cad Saude Publica 2009; 25(11):2455-2466.

19. Traebert EE. Câncer de mama em mulheres idosas: estudo de probabilidade de sobrevida livre de doença [dissertação]. Florianópolis: Universidade Federal de Santa Catarina; 2006.

20. Schneider IJC, D’orsi E. Sobrevida em cinco anos e fatores prognósticos em mulheres com câncer de mama em Santa Catarina, Brasil. Cad Saude Publica 2009; 25(6):1285-1296

21. Ayala ALM. Sobrevida de mulheres com câncer de mama, de uma cidade no sul do Brasil. Rev Bras Enferm 2012; 65(4):566-570.

22. American Cancer Society. Cancer facts \& figures 2008. Atlanta: American Cancer Society; 2008.

23. Schneider IJC. Estudo de sobrevida em mulheres com câncer de mama em Santa Catarina [dissertação]. Florianópolis: Universidade Federal de Santa Catarina; 2008.

24. Rebelo MS. Análise sobre classe social e fatores assistenciais como prognóstico para sobrevida de pacientes com câncer de mama feminina, residentes no Município do Rio de Janeiro, atendidas no Instituto Nacional de Câncer [tese]. Rio de Janeiro: Instituto Nacional de Câncer; 2004.

25. Thuler LCS, Mendonça GA. Estadiamento inicia dos casos de câncer de mama e colo do útero em mulheres brasileiras. Rev Bras Ginecol Obstet 2005; 27(11):656-660

26. Smigal C, Jemal A, Ward E, Cokkinides V, Smith R Howe HL, Thun M. Trends in breast cancer incidence by race and ethnicity: update 2006. CA Cancer J Cin 2006; 56(3):168-183.

27. Novaes HMD, Braga PE, Schout D. Fatores associados à realização de exames preventivos para câncer nas mulheres brasileiras, PNAD 2003. Cien Saude Colet 2006; 11(4):1023-1035.

28. Felden JBB, Figueiredo ACL. Distribuição da gordura corporal e câncer de mama: um estudo de caso-controle no Sul do Brasil. Cien Saude Colet 2011; 16(5):2425-2433

29. Houterman S, Janssen-Heijnen M, Verheij C, Louwman W, Vreugdenhil G, Van der Sangen M, Coebergh JW. Comorbidity has negligible impact on treatment and complications but influences survival in breast cancer patients. Br J Cancer 2004 90(12):2332-2337.
30. Ahern TP, Lash TL, Thwin SS, Silliman RA. Impact of acquired comorbidities on all-cause mortality rates among older breast cancer survivors. Med Care 2009; 47(1):73-79.

31. Mell LK, Dignam JJ, Salama JK, Cohen EEW, Polite BN, Dandekar V, Bhate AD, Witt ME, Haraf DJ, Mittal BB, Vokes EE, Weichselbaum RR. Predictors of competing mortality in advanced head and neck cancer. JCO 2010; 28(1):15-20.

32. Gupta D, Grutsch JF, Lis CG. Patient satisfaction with quality of life as a prognostic indicator in ovarian cancer patients treated in an integrative treatment setting. J Soc Integr Oncol 2008; 6(3):98-104.

33. Lis CG, Gupta D, Grutsch JF. Patient satisfaction with quality of life as a predictor of survival in pancreatic cancer. Int J Gastrointest Cancer 2006; 37(1): $35-44$

34. Lis CG, Gupta D, Granick J, Grutsch JF. Can patient satisfaction with quality of life predict survival in advanced colorectal cancer? Support Care Cancer 2006; 14(11):1104-1110.

35. Gupta D, Granick J, Grutsch JF, Lis CG. The prognostic association of health-related quality of life scores with survival in breast cancer. Support Care Cancer 2007; 15(4):387-393.

36. Tutt A, Ross G. Much still to learn about relations between tumour biology, prognosis, and treatment outcome in early breast cancer. BMG 2000; 320(7233):478-479.

37. Eifel P, Axelson JA, Costa J, Crowley J, Curran Junior WJ, Deshler A, Fulton S, Hendricks CB, Kemeny $M$, Kornblith AB, Louis TA, Markman M, Mayer R, Roter D. National Institutes of Health Consensus Development Conference Statement: adjuvant therapy for breast cancer, November 1-3, 2000. J Natl Cancer Inst 2001; 93(13):979-989.

38. Bezerra KB, Silva DSM, Chein MBC, Ferreira PR, Maranhão JKP, Ribeiro NL, Mochel EG. Qualidade de vida de mulheres tratadas de câncer de mama em uma cidade do nordeste do Brasil. Cien Saude Colet 2013; 18(7):1933-1941.

39. Brito C, Portela MC, Vasconcellos MTL. Avaliação da concordância de dados clínicos e demográficos entre autorizações de procedimento de alta complexidade oncológica e prontuários de mulheres atendidas pelo Sistema Único de Saúde no Estado do Rio de Janeiro, Brasil. Cad Saude Publica 2005; 21(6):1829-1835.

40. MacKinnon JA, Duncan RC, Huang Y, Lee DJ, Fleming LE, Voti L, Rudolph M, Wilkinson JD. Detecting an association between socioeconomic status and late stage breast cancer using spatial analysis and area-based measures. Cancer Epidemiol Biomarkers Prev 2007; 16(4):756-762.

41. Ward E, Jemal A, Cokkinides V, Singh GK, Cardinez C, Ghafoor A, Thun M. Cancer disparities by race/ethnicity and socioeconomic status. CA Cancer J Clin 2004; 54(2):78-93.

42. Woods LM, Rachet B, Coleman MP. Origins of socio-economic inequalities in cancer survival: a review. Ann Oncol 2006; 17(1):5-19. 
43. Byers TE, Wolf HJ, Bauer KR, Bolick-Aldrich S, Chen VW, Finch JL, Fulton JP, Schymura MJ, Shen T, Van Heest S, Yin X; Patterns of Care Study Group. The impact of socioeconomic status on survival after cancer in the United States: findings from the National Program of Cancer Registries Patterns of Care Study. Cancer 2008; 113(3):582-591.

Artigo apresentado em 03/04/2013

Aprovado em 11/08/2013

Versão final apresentada em 26/08/2013 